\title{
On the applicability of minimum channel length criterion for roll-waves in mud-flows
}

\author{
Cristiana Di Cristo ${ }^{1}$, Michele Iervolino ${ }^{2}$, Andrea Vacca ${ }^{3 *}$ \\ ${ }^{1}$ Dipartimento di Ingegneria Civile e Meccanica - Università di Cassino e del Lazio Meridionale, Via Di Biasio 43, 03043 Cassino (FR), \\ Italy. E-mail: dicristo@unicas.it \\ ${ }^{2}$ Dipartimento di Ingegneria Civile, Design, Edilizia, Ambiente, Seconda Università di Napoli, Via Roma 29, 81031 Aversa (CE), Italy. \\ E-mail: michele.iervolino@unina2.it \\ ${ }^{3}$ Dipartimento di Ingegneria Civile, Design, Edilizia, Ambiente, Seconda Università di Napoli, Via Roma 29, 81031 Aversa (CE), Italy. \\ *Corresponding author. Tel.: +39081 5010344.Fax+390815037370. E-mail: vacca@unina.it
}

\begin{abstract}
The paper addresses the prediction of roll-waves occurrence in mud-flows. The spatial growth of a point-wise disturbance is analytically described, based on the linearized flow model of a Herschel and Bulkley fluid, in the neighborhood of an initial uniform base condition. The theoretical achievements allow to generalize to mud-flows the minimum channel criterion commonly used for the prediction of roll-waves in clear-water. The applicability of the criterion is discussed through the comparison with literature laboratory data concerning unstable flows without rollwaves.
\end{abstract}

Keywords: Herschel and Bulkley; Mud-flows; Stability analysis; Roll-waves.

\section{INTRODUCTION}

Mud-flows may produce catastrophic effects due to the significant transport of sediment carried by water and to the occurrence of roll-wave and surge trains. Different fluid mechanic models have been proposed for studying these flows, either based on two-phase approaches (Greco et al., 2012; Iverson, 1997; Jackson, 2000; Pitman and Le, 2005) or on a single-phase description of the flow rheology. Within the latter category, which will be considered herein, the literature offers several formulations: linear and non-linear viscoplastic models (Huang and Garcia, 1997, 1998; Johnson, 1970; Liu and Mei, 1989; O'Brien and Julien, 1988), dilatant fluid models (Bagnold, 1954; Mainali and Rajaratnam, 1994; Takahashi, 1978) biviscous modified Bingham models (Dent and Lang, 1983). Viscoplastic models (i.e.: Herschel and Bulkley fluids) are the most widely used to describe the rheology of mud suspensions (Huang and Garcia, 1998; Jiang and LeBlond, 1992; Liu and Mei, 1989), which usually exhibit a yield stress below which they are almost rigid and above which they can flow. Moreover, because of their high apparent viscosity, natural flows of concentrated suspension are laminar. Different formulations have been presented such as Bingham (Huang and Garcia, 1997) or Herschel and Bulkley (see for instance Balmforth and Liu, 2004; Coussot, 1997; Fernández-Nieto et al., 2010; Huang and Garcia, 1998) models for fluids in which the yield stress is important, conversely its effect is not considered in the powerlaw formulation, more appropriate and convenient for fluids with a shear-thinning behavior ( $\mathrm{Ng}$ and $\mathrm{Mei}, 1994)$. In this paper, among the available Herschel and Bulkley formulations, i.e. three-equation (Balmforth and Liu, 2004; Di Cristo et al., 2013d; Huang and Garcia, 1998) or two-equation (Ancey, 2001, 2005; Ancey et al., 2012; Coussot, 1997; Laigle and Coussot, 1997) models, the latter, which is more usually adopted in engineering applications, is considered.

Despite the different rheology, the mechanism of spatial development of roll-waves in mud and clear-water flows exhibits strong similarities (Takahashi, 1991). As the wave develops in the downstream direction, three different phases may be observed. In the first one, roll-waves amplitude exponentially increases in the streamwise direction; in the second phase, waves begin to overtake each other, increasing their period. Finally, in the third phase waves are characterized by steep downstream fronts, and by a further progressive increase of their mean period. From the theoretical point of view and with reference to the first phase, for both newtonian and non-newtonian fluids, the roll waves development has been related to the existence of linearly unstable conditions of depthaveraged one-dimensional models.

As far as the water is concerned, normal mode analysis applied to linearized St. Venant equations, starting form an initial uniform condition, allowed to individuate the limit values of the Froude and Reynolds numbers above which the perturbations may grow (Berlamont, 1976; Berlamont and Vanderstappen, 1981; Brock, 1969; Ponce and Simon, 1977). However, the overcoming of the marginal stability condition, which for clear-water occurs in supercritical flows, has been shown to be only a necessary condition for the roll-waves occurrence, since a minimum channel length is necessary to allow the growth of the perturbation up to a perceivable level. Indeed, based on the results of spatial linear analysis (Di Cristo and Vacca, 2005; Di Cristo et al., 2012; Julien and Hartley, 1986; Liggett, 1975; Vedernikov, 1946), criteria for the prediction of roll waves occurrence, known as minimum channel length criteria, have been proposed for water flows in turbulent (Di Cristo et al., 2008, 2010; Montuori, 1963) and laminar (Julien and Hartley, 1986) regimes and for dense granular flows (Di Cristo et al., 2009).

Linear stability analysis, carried out perturbing an uniform base flow, has been also widely applied to mud-flows in the one-dimensional shallow-water framework. With reference to power-law fluids, $\mathrm{Ng}$ and Mei (1994) individuated the flow stability conditions of the linearized mass and momentum conservation equations. The results of $\mathrm{Ng}$ and Mei (1994) have been generalized accounting for a surface tension (Hwang et al., 1994), considering a porous bottom (Di Cristo et al., 2013c; Pascal, 2006) and including a superficial shear stress effect (Pascal and D'Alessio, 2007). In presence of yield stress and with reference to the two-equation model, linear stability conditions have been found by Trowbridge (1987) for Bingham 
fluids and by Coussot (1994) for Herschel and Bulkley fluids. The wave dynamics of a Herschel and Bulkley fluid in both stable and unstable condition has been investigated by Di Cristo et al. (2013a,b), proving the convective character of the instability which ensures that an initial disturbance, localized in space, does not spread in both downstream and upstream directions (absolute instability) but it is swept away from the source along a preferential direction (Huerre and Monkewitz, 1990). However, similarly to the clear-water case, experimental evidences (Coussot, 1994; Zanuttigh and Lamberti, 2007) showed that, even in linearly unstable conditions, roll-waves may not appear. Coussot (1994) ascribed this inconsistency between theory and experiments to the potential role of the limited channel length, as observed for Newtonian laminar flows (Julien and Hartley, 1986).

The objective of the presented analysis is to apply the minimum channel length criterion for the prediction of the rollwaves to the case of mud-flows, and to verify if it can explain the observed discrepancy between the linear analysis results and the experimental observations. In particular, in the paper, generalizing the results for clear-water, the expression of the spatial growth rate of a pointwise instantaneous disturbance, superposed to an Herschel and Bulkley fluid uniform flow, is deduced. Then, on the basis of such a theoretical achievement the minimum channel length criterion for the prediction of rollwaves is applied.

\section{GOVERNING EQUATIONS}

Let us consider a homogeneous medium flowing over a fixed bed without lateral inflow or outflow. The density of the flowing material and the bed slope are assumed to be constant. In what follows the curvature effects have been neglected (Di Nucci et al., 2007, 2011). Moreover, supposing that:

- spatial variations occur over scales larger than flow depth;

- flow resistance by the sidewalls is negligible with respect to that by bottom;

- momentum correction factor is equal to unity;

- surface tension is negligible;

the dimensional depth-averaged momentum and mass conservation equations are (Ancey et al., 2012)

$$
\begin{aligned}
& \frac{\partial \tilde{h} \tilde{u}}{\partial \tilde{t}}+\frac{\partial \tilde{h} \tilde{u}^{2}}{\partial \tilde{x}}+g \tilde{h} \cos \theta \frac{\partial \tilde{h}}{\partial \tilde{x}}-g \tilde{h} \sin \theta+\frac{\tilde{\tau}_{b}}{\rho}=0, \\
& \frac{\partial \tilde{h}}{\partial \tilde{t}}+\tilde{u} \frac{\partial \tilde{h}}{\partial \tilde{x}}+\tilde{h} \frac{\partial \tilde{u}}{\partial \tilde{x}}=0,
\end{aligned}
$$

where $\tilde{x}$ is the streamwise coordinate, $\tilde{t}$ is the time, $g$ and $\rho$ are the gravity and the fluid density, respectively, $\tilde{h}$ is the flow depth, $\theta$ is the bed inclination angle with respect to the horizontal plane, $\tilde{\tau}_{b}$ is the shear stress at the bottom, $\tilde{u}$ is the depth-averaged velocity:

$$
\tilde{u}=\frac{1}{\tilde{h}} \int_{0}^{\tilde{h}} \tilde{U}(\tilde{z}) d \tilde{z}
$$

For the Herschel and Bulkley flow model, the shear stress at the bottom $\left(\tilde{\tau}_{b}\right)$ is expressed by the following relation (Ancey et al., 2012):

$$
\left(\frac{\mu_{n}}{\tilde{\tau}_{c}}\right)^{1 / n} \frac{2 n+1}{n} \frac{\tilde{u}}{\tilde{h}}=\left(\frac{\tilde{\tau}_{b}}{\tilde{\tau}_{c}}\right)^{1 / n}\left(1-\frac{\tilde{\tau}_{c}}{\tilde{\tau}_{b}}\right)^{1+1 / n}\left(1+\frac{n}{n+1} \frac{\tilde{\tau}_{c}}{\tilde{\tau}_{b}}\right),
$$

where $\tilde{\tau}_{c}$ is the yield stress; $n$ is the rheological index, ranging between 0 and 1 for a shear-thinning fluid (Bird et al., 1983); $\mu_{n}$ is the viscosity coefficient, named also consistency. The special limit of $n=1$ corresponds to a Newtonian fluid and $\mu_{l}=\mu$ becomes the ordinary dynamic viscosity.

In uniform flow condition (Fig. 1), denoted by the subscript 0 , the following relation holds:

$$
\left(\frac{\mu_{n}}{\tilde{\tau}_{c}}\right)^{1 / n} \frac{2 n+1}{n} \frac{\tilde{u}_{0}}{\tilde{h}_{0}}=\left(\frac{\tilde{\tau}_{0}}{\tilde{\tau}_{c}}\right)^{1 / n}\left(1-\frac{\tilde{\tau}_{c}}{\tilde{\tau}_{0}}\right)^{1+1 / n}\left(1+\frac{n}{n+1} \frac{\tilde{\tau}_{c}}{\tilde{\tau}_{0}}\right)
$$

with $\tilde{\tau}_{0}=\rho g \tilde{h}_{0} \sin \theta$ the bottom shear stress. As shown in Fig. 1 , the flow is divided into two regions by a yield interface: the plug layer, where the local velocity $\tilde{U}_{0}$ is constant, and the yield layer, where the shear stress exceeds the yield stress.

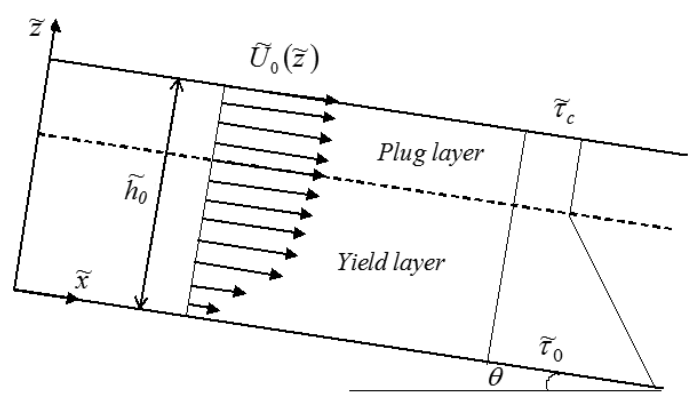

Fig. 1. A sketch of the viscoplastic fluid flowing over a fixed inclined bed in uniform flow condition.

Introducing the following dimensionless quantities:

$$
\begin{aligned}
x & =\tilde{x} / \tilde{h}_{0}, t=\tilde{t} \tilde{u}_{0} / \tilde{h}_{0}, u=\tilde{u} / \tilde{u}_{0}, h=\tilde{h} / \tilde{h}_{0}, \\
\tau & =\tilde{\tau} / \tilde{\tau}_{0}
\end{aligned}
$$

and accounting for (5), (1)-(2) and (4) can be rewritten in the following dimensionless form:

$$
\begin{aligned}
& \frac{\partial h u}{\partial t}+\frac{\partial h u^{2}}{\partial x}+\frac{h}{F_{r}^{2}} \frac{\partial h}{\partial x}=-\frac{h}{\operatorname{Re}} f_{1}(n, \chi)\left(\frac{\tau_{b}}{h}-1\right), \\
& \frac{\partial h}{\partial t}+u \frac{\partial h}{\partial x}+h \frac{\partial u}{\partial x}=0,
\end{aligned}
$$

where $\widetilde{U}$ denotes the local velocity. 


$$
\begin{aligned}
& \left(\frac{F_{r}^{2}}{\tau_{c} \operatorname{Re} \tan \theta}\right)^{1 / n} \frac{2 n+1}{n} \frac{u}{h}=\left(\frac{\tau_{b}}{\tau_{c}}\right)^{1 / n} \\
& \left(1-\frac{\tau_{c}}{\tau_{b}}\right)^{1+1 / n}\left(1+\frac{n}{n+1} \frac{\tau_{c}}{\tau_{b}}\right)
\end{aligned}
$$

with:

$f_{1}(n, \chi)=\left[\frac{(2 n+1)(n+1)}{n(1+n+n \chi)}\right]^{n} \frac{1}{(1-\chi)^{n+1}}$,

$\chi=\tau_{c} / \tau_{0}$ is the yield parameter always less then unity, since for Herschel and Bulkley fluid no flow occurs unless $\tau_{0}>\tau_{c}$. In (7)-(8) $\quad F_{r}=u_{0} / \sqrt{g h_{0} \cos \theta}$ and $R e=\rho u_{0}^{2-n} h_{0}^{n} / \mu_{n}$ are the Froude and the generalized Reynolds numbers, respectively. Depending on the value of the Froude number, subcritical $\left(F_{r}<\right.$ $1)$, critical $\left(F_{r}=1\right)$ or supercritical $\left(F_{r}>1\right)$ conditions of the base uniform flow may occur.

\section{DISTURBANCE SPATIAL GROWTH}

The hyperbolic character of the system (7)-(8), along with the convective character of the instability (Di Cristo et al., 2013a), suggest to characterize the spatial evolution of a perturbation through the study of the time-asymptotic solution of the linearized version of system (7)-(8), under the action of an impulsive pointwise disturbance. Following a procedure similar to that used by Liggett (1975) for the St. Venant equations, assuming an unbounded domain and considering as initial condition an uniform one, we rewrite the system (7)-(8) in the variables:

$$
\xi=x, \quad \eta=c_{+} t-x
$$

with $c_{+}=1+1 / F$. Expanding the unknown functions $u$ and $h$ in series around $\eta=0$ :

$$
\begin{aligned}
& u=1+u_{1}(\xi) \eta+u_{2}(\xi) \eta^{2}+\ldots \\
& h=1+h_{1}(\xi) \eta+h_{2}(\xi) \eta^{2}+\ldots
\end{aligned}
$$

and approximating the right hand side of (7) as:

$$
\begin{aligned}
& \frac{f_{1}(n, \chi)}{\operatorname{Re}}\left(\frac{\tau_{b}}{h}-1\right)=\frac{f_{1}(n, \chi)}{\operatorname{Re}} \\
& {\left[\left.\frac{\partial}{\partial h} \frac{\tau_{b}}{h}\right|_{0} h_{1} \tau+\left.\frac{\partial}{\partial u} \frac{\tau_{b}}{h}\right|_{0} u_{1} \tau+\ldots\right]}
\end{aligned}
$$

the following linear system is deduced:

$$
\begin{aligned}
& \left(1+\frac{1}{F_{r}}\right)\left(u_{1}+2 u_{2} \eta\right)+\left(1+u_{1}+2 u_{2} \eta^{2}\right)\left(\frac{d u_{1}}{d \xi} \eta-u_{1}-2 u_{2} \eta\right) \\
& +\frac{1}{F_{r}^{2}}\left(\frac{d h_{1}}{d \xi} \eta-h_{1}-2 h_{2} \eta\right)= \\
& =-\frac{f_{1}(n, \chi)}{\operatorname{Re}}\left(\left.\frac{\partial}{\partial h} \frac{\tau_{b}}{h}\right|_{0} h_{1} \eta+\left.\frac{\partial}{\partial u} \frac{\tau_{b}}{h}\right|_{0} u_{1} \eta\right), \\
& \left(1+\frac{1}{F_{r}}\right)\left(h_{1}+2 h_{2} \eta\right)+\left(1+u_{1}+2 u_{2} \eta^{2}\right)\left(\frac{d h_{1}}{d \xi} \eta-h_{1}-2 h_{2} \eta\right) \\
& +\left(1+h_{1} \eta+2 h_{2} \eta^{2}\right)\left(\frac{d u_{1}}{d \xi} \eta-u_{1}-2 u_{2} \eta\right)=0 .
\end{aligned}
$$

At order of $\eta$ the system (15)-(16) leads to the following differential equation:

$$
\frac{d h_{1}}{d \xi}=\vartheta h_{1}^{2}-\gamma h_{1}
$$

where

$$
\begin{aligned}
& \vartheta=\frac{3}{2\left(F_{r}+1\right)}, \\
& \gamma=\frac{f_{1}(n, \chi)}{2 \operatorname{Re}} \frac{F_{r}^{2}}{F_{r}+1}\left(\left.\frac{1}{F_{r}} \frac{\partial}{\partial u} \frac{\tau_{b}}{h}\right|_{0}+\left.\frac{\partial}{\partial h} \frac{\tau_{b}}{h}\right|_{0}\right) .
\end{aligned}
$$

The solution of the differential Eq. (17), in the initial development phase, can be approximated as (Liggett, 1975):

$$
h_{1}=\kappa e^{\gamma \xi}
$$

being $\kappa$ an integration constant.

With reference to (9), it is easy to verify that the following relations hold:

$$
\begin{aligned}
& \left.\frac{\partial}{\partial u} \frac{\tau_{b}}{h}\right|_{0}=-\frac{f_{2}(n, \chi)}{f_{1}(n, \chi)}, \\
& \left.\frac{\partial}{\partial h} \frac{\tau_{b}}{h}\right|_{0}=\frac{f_{3}(n, \chi)-f_{2}(n, \chi)}{f_{1}(n, \chi)},
\end{aligned}
$$

where the expressions of the positive functions $f_{2}(n, \chi)$ and $f_{3}(n, \chi)$ - with $f_{3}(n, \chi)>f_{2}(n, \chi)-$ are:

$f_{2}(n, \chi)=n \frac{(1-\chi)(n+n \chi+1)}{n+1+2 n \chi+2 n^{2} \chi^{2}}$

$\left[\frac{(2 n+1)(n+1)}{n(1+n+n \chi)}\right]^{n} \frac{1}{(1-\chi)^{n+1}}$, 


$$
\begin{aligned}
& f_{3}(n, \chi)=\left[1+2 n \frac{(1-\chi)(n+n \chi+1)}{n+1+2 n \chi+2 n^{2} \chi^{2}}\right] \\
& {\left[\frac{(2 n+1)(n+1)}{n(1+n+n \chi)}\right]^{n} \frac{1}{(1-\chi)^{n+1}} .}
\end{aligned}
$$

Accounting for (20)-(23) and defining $1 / F_{r^{*}}=f_{3}(n, \chi) /$ $f_{2}(n, \chi)-1$, the $\gamma$ coefficient can be alternatively rewritten as:

$\gamma=\frac{f_{2}(n, \chi)}{2 \operatorname{Re}} \frac{F_{r}}{F_{r}+1}\left(\frac{F_{r}}{F_{r^{*}}}-1\right)$.

Thus, Eq. (19) expresses the exponential growth of the flow depth perturbation along $\xi$, provided that $\gamma>0$, which according to Eq. (24) is equivalent to $F_{r}>F_{r^{*}}$. It is easy to verify that $F_{r^{*}}$ represents the marginal stability limit deduced by Coussot (1994), owing to the convective character of the instability (Di Cristo et al., 2013a). Finally it is worth of note that for shear-thinning fluids the marginal stability condition occurs always in hypocritical condition of flow, i.e. $F_{r^{*}}<1$.

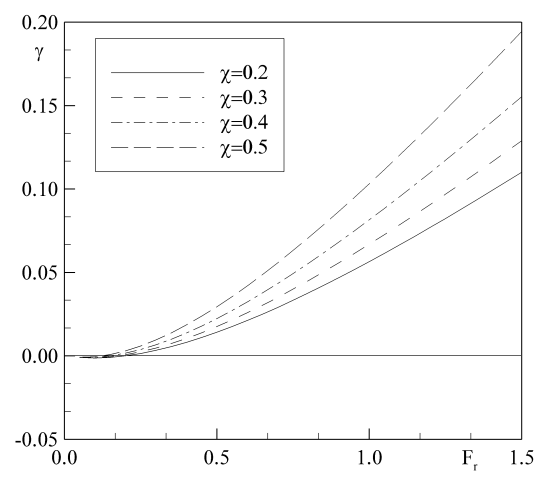

Fig. 2. Spatial growth-rate as a function of $F_{r}$ for $n=0.33(R e=$ $10)$.

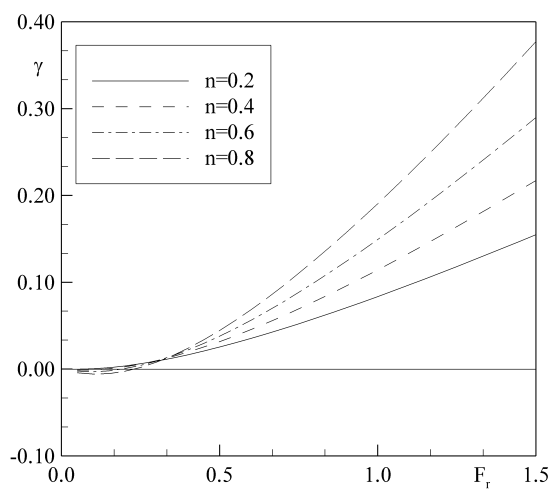

Fig. 3. Spatial growth-rate as a function of $F_{r}$ for $\chi=0.5(R e=10)$.

The dependence of the spatial growth rate on the Reynolds and Froude numbers can easily be deduced from Eq. (24), i.e. $\gamma$ decreases with $R e$ and attains a local minimum at $\hat{F}_{r}=\sqrt{F_{r^{*}}+1}-1$, while the influence of the rheological parameters is not so evident. For a fixed value of Reynolds number $(R e=10)$, Figs. 2 and 3 show the spatial growth rate as a function of the Froude number for $n=1 / 3$ and different $\chi$ values and for $\chi=0.5$ and different $n$ values, respectively.
Negative spatial growth-rates correspond to stable conditions $\left(F_{r}<F_{r^{*}}\right)$, while positive ones witness the occurrence of unstability $\left(F_{r}>F_{r^{*}}\right)$.

For sake of definiteness, in Table 1 the $F_{r^{*}}$ values, for each of the considered cases, are reported. Figs. 2 and 3 show that while an increase of dimensionless yield stress induces larger values of $\gamma$, the dependence of the spatial growth-rate on the rheological exponent $n$ is not monotone.

Table 1. Froude numbers corresponding to marginal stability condition.

\begin{tabular}{cccc}
\hline \multicolumn{2}{c}{$\chi=0.5$} & \multicolumn{2}{c}{$n=1 / 3$} \\
$n$ & $F_{r^{*}}$ & $\chi$ & $F_{r^{*}}$ \\
\hline 0.20 & 0.084 & 0.20 & 0.200 \\
0.40 & 0.145 & 0.30 & 0.176 \\
0.60 & 0.193 & 0.40 & 0.151 \\
0.80 & 0.232 & 0.50 & 0.126 \\
\hline
\end{tabular}

\section{MINIMUM CHANNEL LENGTH CRITERION FOR MUD-FLOWS}

The expression of the spatial growth rate deduced in the previous section is used to formulate a minimum channel length criterion for mud-flows. This criterion is successively applied to analyze the unstable flows without roll-waves experimentally observed by Coussot (1994).

Table 2a. Flow and fluid characteristics of mud-flow tests with

\begin{tabular}{|c|c|c|c|c|c|c|}
\hline Test & $\begin{array}{c}S \\
(\%)\end{array}$ & $\begin{array}{c}Q \\
(1 / \mathrm{s})\end{array}$ & $\begin{array}{c}\mu_{n} \\
\left(\mathrm{~kg} / \mathrm{m} / \mathrm{s}^{2-\mathrm{n}}\right)\end{array}$ & $\begin{array}{c}\tilde{\tau}_{c} \\
(\mathrm{~Pa})\end{array}$ & $\begin{array}{l}C_{\mathrm{v}} \\
(-)\end{array}$ & $\begin{array}{c}\tilde{h}_{0} \\
(\mathrm{~m})\end{array}$ \\
\hline 1 & 38.9 & 1.7 & 5.7 & 16.8 & 0.214 & 0.010 \\
\hline 2 & 14.0 & 3.2 & 5.3 & 14.4 & 0.211 & 0.021 \\
\hline 3 & 35.2 & 1.5 & 12.3 & 30.0 & 0.244 & 0.017 \\
\hline 4 & 32.4 & 1.5 & 12.3 & 30.0 & 0.244 & 0.018 \\
\hline 5 & 24.7 & 1.3 & 5.7 & 16.8 & 0.214 & 0.013 \\
\hline 6 & 14.9 & 2.0 & 5.3 & 14.4 & 0.211 & 0.019 \\
\hline 7 & 29.1 & 1.5 & 12.3 & 30.0 & 0.244 & 0.019 \\
\hline 8 & 22.3 & 2.5 & 12.3 & 30.0 & 0.244 & 0.025 \\
\hline 9 & 32.4 & 1.0 & 12.3 & 30.0 & 0.244 & 0.017 \\
\hline 10 & 17.9 & 2.8 & 5.2 & 17.4 & 0.218 & 0.018 \\
\hline 11 & 25.7 & 1.5 & 12.3 & 30.0 & 0.244 & 0.021 \\
\hline 12 & 19.9 & 2.5 & 12.3 & 30.0 & 0.244 & 0.027 \\
\hline 13 & 38.9 & 0.6 & 12.3 & 30.0 & 0.244 & 0.014 \\
\hline 14 & 16.9 & 2.8 & 5.2 & 17.4 & 0.218 & 0.019 \\
\hline 15 & 17.9 & 6.3 & 4.2 & 18.6 & 0.229 & 0.019 \\
\hline 16 & 18.9 & 2.0 & 7.5 & 21.6 & 0.238 & 0.020 \\
\hline 17 & 15.9 & 2.8 & 5.2 & 17.4 & 0.218 & 0.020 \\
\hline 18 & 14.0 & 3.3 & 5.2 & 17.4 & 0.218 & 0.022 \\
\hline 19 & 14.9 & 2.8 & 5.2 & 17.4 & 0.218 & 0.020 \\
\hline 20 & 21.3 & 1.2 & 7.5 & 21.6 & 0.238 & 0.017 \\
\hline 21 & 35.2 & 0.5 & 12.3 & 30.0 & 0.244 & 0.014 \\
\hline 22 & 13.0 & 7.7 & 4.2 & 18.6 & 0.229 & 0.024 \\
\hline 23 & 15.9 & 4.3 & 4.2 & 18.6 & 0.229 & 0.019 \\
\hline 24 & 17.9 & 3.3 & 4.2 & 18.6 & 0.229 & 0.017 \\
\hline 25 & 13.0 & 6.3 & 4.8 & 20.4 & 0.240 & 0.026 \\
\hline 26 & 14.0 & 5.3 & 4.8 & 20.4 & 0.240 & 0.024 \\
\hline 27 & 14.0 & 4.5 & 4.2 & 18.6 & 0.229 & 0.021 \\
\hline 28 & 12.0 & 4.8 & 4.8 & 20.4 & 0.240 & 0.026 \\
\hline 29 & 11.5 & 4.8 & 4.8 & 20.4 & 0.240 & 0.027 \\
\hline 30 & 19.9 & 2.2 & 15.0 & 39.0 & 0.254 & 0.031 \\
\hline 31 & 17.4 & 2.6 & 15.0 & 39.0 & 0.254 & 0.035 \\
\hline 32 & 13.0 & 4.1 & 15.0 & 39.0 & 0.254 & 0.047 \\
\hline 33 & 14.0 & 3.0 & 15.0 & 39.0 & 0.254 & 0.043 \\
\hline
\end{tabular}
roll-waves. 
Table 2b. Flow and fluid characteristics of mud-flow tests without roll-waves.

\begin{tabular}{ccccccc}
\hline Test & $\begin{array}{c}S \\
(\%)\end{array}$ & $\begin{array}{c}Q \\
(\mathrm{l} / \mathrm{s})\end{array}$ & $\begin{array}{c}\mu_{n} \\
\left(\mathrm{~kg} / \mathrm{m} / \mathrm{s}^{2-\mathrm{n}}\right)\end{array}$ & $\begin{array}{c}\tilde{\tau}_{c} \\
(\mathrm{~Pa})\end{array}$ & $\begin{array}{c}C_{\mathrm{v}} \\
(-)\end{array}$ & $\begin{array}{c}\tilde{h}_{0} \\
(\mathrm{~m})\end{array}$ \\
\hline 1 & 23.7 & 2.0 & 12.3 & 30.0 & 0.244 & 0.023 \\
2 & 20.8 & 2.2 & 12.3 & 30.0 & 0.244 & 0.026 \\
3 & 19.9 & 1.4 & 4.5 & 16.0 & 0.215 & 0.014 \\
4 & 13.0 & 3.9 & 4.5 & 16.0 & 0.215 & 0.022 \\
5 & 19.9 & 1.4 & 5.2 & 17.4 & 0.218 & 0.015 \\
6 & 13.0 & 3.9 & 5.2 & 17.4 & 0.218 & 0.024 \\
7 & 21.8 & 0.4 & 4.5 & 13.7 & 0.205 & 0.011 \\
8 & 20.8 & 1.4 & 12.3 & 30.0 & 0.244 & 0.024 \\
9 & 19.1 & 1.4 & 12.3 & 30.0 & 0.244 & 0.026 \\
10 & 19.9 & 0.4 & 4.5 & 13.7 & 0.205 & 0.011 \\
11 & 14.4 & 1.7 & 5.2 & 17.4 & 0.218 & 0.020 \\
12 & 18.9 & 0.9 & 7.5 & 21.6 & 0.238 & 0.018 \\
13 & 13.0 & 2.0 & 4.5 & 16.0 & 0.215 & 0.020 \\
14 & 13.0 & 2.0 & 5.2 & 17.4 & 0.218 & 0.022 \\
15 & 18.9 & 0.9 & 9.3 & 26.4 & 0.264 & 0.021 \\
16 & 34.3 & 0.8 & 16.8 & 48.0 & 0.271 & 0.020 \\
17 & 10.5 & 1.2 & 5.7 & 16.8 & 0.214 & 0.025 \\
18 & 9.0 & 3.1 & 4.5 & 16.0 & 0.215 & 0.028 \\
19 & 9.0 & 3.1 & 5.2 & 17.4 & 0.218 & 0.031 \\
20 & 11.0 & 2.5 & 12.3 & 30.0 & 0.244 & 0.042 \\
21 & 10.5 & 3.3 & 12.8 & 32.4 & 0.245 & 0.047 \\
22 & 20.4 & 2.0 & 17.8 & 52.8 & 0.265 & 0.036 \\
\hline
\end{tabular}

Referring to a wide rectangular channel of length $L$, the minimum channel length criterion for clear-water relies on the comparison between the dimensionless channel length (Montuori number):

$M=S g L / u_{0}^{2}$

with $S=\sin \theta$ the bed slope and the following limiting value:

$M_{\min }=\frac{\varepsilon}{\gamma} \frac{S}{F_{r}^{2} \cos (\theta)}$,

where $\gamma$ is the spatial growth-rate factor of an impulsive pointwise disturbance to the linearized St. Venant equations. According to this criterion, roll-waves appear whenever $M>M_{\min }$. The value of the dimensionless coefficient $\varepsilon$ was estimated by best-fitting available field and laboratory data of water flows in both laminar and turbulent conditions (Julien and Hartley, 1986; Liggett, 1975; Montuori, 1963).

The above criterion is herein applied to the mud-flow laboratory experiments carried out by Coussot (1994) in a 8m long and $0.6 \mathrm{~m}$ wide rectangular channel. To comply with our hypothesis of wide channel, only the experiments with depthto-width ratio $\tilde{h}_{0} / B<1 / 10$ have been selected, leading to a data-set of 55 tests: 33 with roll-waves, 22 without roll-waves.

Table 3a. Dimensionless numbers of mud-flow tests with roll-waves.

\begin{tabular}{|c|c|c|c|c|c|c|c|}
\hline Test & $\chi$ & $R e$ & $F_{r}$ & $F_{r^{*}}$ & $M$ & $M_{\min }$ & $M_{\min } / M$ \\
\hline 1 & 0.33 & 6.36 & 0.97 & 0.17 & 367 & 15 & 0.04 \\
\hline 2 & 0.38 & 7.16 & 0.57 & 0.16 & 167 & 40 & 0.24 \\
\hline 3 & 0.38 & 1.23 & 0.38 & 0.16 & 1220 & 95 & 0.08 \\
\hline 4 & 0.38 & 1.13 & 0.35 & 0.16 & 1268 & 119 & 0.09 \\
\hline 5 & 0.39 & 2.76 & 0.47 & 0.15 & 712 & 60 & 0.08 \\
\hline 6 & 0.39 & 3.81 & 0.42 & 0.15 & 365 & 75 & 0.20 \\
\hline 7 & 0.40 & 1.02 & 0.31 & 0.15 & 1333 & 163 & 0.12 \\
\hline 8 & 0.40 & 1.68 & 0.34 & 0.15 & 622 & 122 & 0.20 \\
\hline 9 & 0.41 & 0.62 & 0.25 & 0.15 & 2561 & 291 & 0.11 \\
\hline 10 & 0.41 & 7.21 & 0.63 & 0.15 & 207 & 33 & 0.16 \\
\hline 11 & 0.41 & 0.90 & 0.27 & 0.15 & 1413 & 239 & 0.17 \\
\hline 12 & 0.41 & 1.50 & 0.30 & 0.15 & 657 & 169 & 0.26 \\
\hline 13 & 0.41 & 0.35 & 0.21 & 0.15 & 5703 & 595 & 0.10 \\
\hline 14 & 0.42 & 6.82 & 0.59 & 0.15 & 213 & 37 & 0.17 \\
\hline 15 & 0.42 & 33.30 & 1.33 & 0.15 & 44 & 9 & 0.20 \\
\hline 16 & 0.42 & 2.50 & 0.38 & 0.15 & 542 & 96 & 0.18 \\
\hline 17 & 0.42 & 6.42 & 0.55 & 0.15 & 219 & 42 & 0.19 \\
\hline 18 & 0.43 & 7.20 & 0.54 & 0.15 & 176 & 43 & 0.25 \\
\hline 19 & 0.43 & 6.03 & 0.51 & 0.14 & 226 & 49 & 0.21 \\
\hline 20 & 0.43 & 1.31 & 0.28 & 0.14 & 1246 & 192 & 0.15 \\
\hline 21 & 0.43 & 0.24 & 0.16 & 0.14 & 8233 & 2824 & 0.34 \\
\hline 22 & 0.44 & 32.61 & 1.09 & 0.14 & 36 & 12 & 0.34 \\
\hline 23 & 0.45 & 16.72 & 0.86 & 0.14 & 91 & 18 & 0.20 \\
\hline 24 & 0.45 & 12.63 & 0.79 & 0.14 & 136 & 21 & 0.15 \\
\hline 25 & 0.45 & 19.34 & 0.83 & 0.14 & 60 & 19 & 0.32 \\
\hline 26 & 0.46 & 16.06 & 0.78 & 0.14 & 78 & 21 & 0.27 \\
\hline 27 & 0.46 & 15.66 & 0.76 & 0.14 & 90 & 22 & 0.25 \\
\hline 28 & 0.48 & 11.84 & 0.60 & 0.13 & 101 & 34 & 0.34 \\
\hline 29 & 0.48 & 11.33 & 0.57 & 0.13 & 103 & 38 & 0.36 \\
\hline 30 & 0.45 & 0.83 & 0.21 & 0.14 & 1140 & 463 & 0.41 \\
\hline 31 & 0.46 & 0.93 & 0.21 & 0.14 & 910 & 482 & 0.53 \\
\hline 32 & 0.47 & 1.37 & 0.22 & 0.14 & 476 & 417 & 0.88 \\
\hline 33 & 0.48 & 0.92 & 0.18 & 0.13 & 794 & 794 & 1.00 \\
\hline
\end{tabular}


On the applicability of minimum channel length criterion for roll-waves in mud-flows

Table 3b. Dimensionless numbers of mud-flow tests without roll-waves.

\begin{tabular}{cccccccc}
\hline Test & $\chi$ & $R e$ & $F_{r}$ & $F_{r^{*}}$ & $M$ & $M_{\min }$ & $M_{\min } / M$ \\
\hline 1 & 0.402 & 1.28 & 0.31 & 0.151 & 875 & 162 & 0.18 \\
2 & 0.410 & 1.32 & 0.29 & 0.149 & 779 & 190 & 0.24 \\
3 & 0.438 & 3.53 & 0.45 & 0.142 & 573 & 64 & 0.11 \\
4 & 0.429 & 10.84 & 0.64 & 0.145 & 117 & 31 & 0.27 \\
5 & 0.437 & 2.74 & 0.40 & 0.143 & 676 & 84 & 0.12 \\
6 & 0.428 & 8.43 & 0.56 & 0.145 & 138 & 40 & 0.29 \\
7 & 0.452 & 0.69 & 0.21 & 0.139 & 3915 & 536 & 0.13 \\
8 & 0.434 & 0.68 & 0.20 & 0.143 & 1698 & 613 & 0.36 \\
9 & 0.444 & 0.62 & 0.18 & 0.141 & 1779 & 943 & 0.52 \\
10 & 0.468 & 0.54 & 0.17 & 0.135 & 4914 & 1209 & 0.24 \\
11 & 0.460 & 2.83 & 0.33 & 0.137 & 531 & 125 & 0.23 \\
12 & 0.462 & 0.77 & 0.20 & 0.136 & 2128 & 600 & 0.28 \\
13 & 0.464 & 4.18 & 0.38 & 0.136 & 357 & 90 & 0.25 \\
14 & 0.462 & 3.25 & 0.34 & 0.136 & 421 & 121 & 0.29 \\
15 & 0.478 & 0.53 & 0.16 & 0.132 & 2792 & 1511 & 0.54 \\
16 & 0.486 & 0.25 & 0.15 & 0.130 & 6116 & 2456 & 0.39 \\
17 & 0.491 & 1.03 & 0.16 & 0.129 & 1259 & 1236 & 0.98 \\
18 & 0.481 & 5.46 & 0.35 & 0.132 & 206 & 105 & 0.51 \\
19 & 0.480 & 4.25 & 0.31 & 0.132 & 243 & 142 & 0.59 \\
20 & 0.474 & 0.83 & 0.15 & 0.133 & 886 & 2261 & 2.55 \\
21 & 0.478 & 1.10 & 0.17 & 0.132 & 599 & 1037 & 1.73 \\
22 & 0.511 & 0.49 & 0.16 & 0.124 & 1899 & 1466 & 0.77 \\
\hline
\end{tabular}

Table $2(\mathrm{a}, \mathrm{b})$ reports the flow conditions $(S=$ bed slope, $Q=$ flow rate) and the fluid characteristics $\left(\mu_{n}=\right.$ consistency, $\tilde{\tau}_{c}=$ yield stress, $C_{v}=$ solid volumetric concentration) of the selected tests. As suggested by Coussot (1994), the rheological exponent is assumed for all tests as $n=1 / 3$, while the value of the depth $\tilde{h}_{0}$ has been estimated considering uniform flow conditions in an infinitely wide channel.

In Table $3(a, b)$ the computed values of the governing dimensionless parameters $\left(\chi, R e, F_{r}, F_{r^{*}}\right)$ are reported for the considered experiments, along with the Montuori number $(M)$, the corresponding theoretical limiting value $\left(M_{\min }\right)$ and their ratio. For the considered tests the linear stability analysis predicts instability, being $F_{r}>F_{r^{*}}$ in all cases. The limiting Montuori number has been evaluated through Eq. (26) with the spatial growth-rate factor expressed by Eq. (24). The value of the dimensionless coefficient $\varepsilon=3.5$ has been chosen as the lowest for which Eq. (26) predicts roll-waves occurrence in all the tests in which they were observed (Table 3a). According to the minimum channel length criterion, roll-waves in unstable conditions of flow are not expected if the channel length is lower than the minimum value, i.e. $M<M_{\min }$. Table $3 \mathrm{~b}$ shows that the minimum channel length criterion supports very partially the experimental evidences, since it explains only the $9 \%$ of the unstable flows observed without roll-waves. In order to verify whether such a result may be attributed to rheometrical uncertainties, we have reworked the experimental data accounting for the potential sources of error in flow curve determination, which, according to Coussot (1994), may lead to a maximum relative error of $20 \%$ of the computed shear stress. Even accounting for this aspect the performance of the criterion improves only up to a maximum of $20 \%$. It may be therefore concluded that rheometrical uncertainties cannot be considered as responsable of the unsatisfactory result.

On the other hand, Tables $3 \mathrm{a}, \mathrm{b}$ indicate that all experimental data refer to subcritical conditions for which the downstream boundary condition may determine a non-negligible influence either on the initial condition, which may differ from the uniform one, or on reflection of the wave at the end of the channel, which in the present theroretical model is completely neglected. Therefore it may be argued that, differentlty from the clear-water case, a minimum channel criterion which does not fully account for the downstream boundary condition may not straightfowardly be applied to mud-flows.

\section{CONCLUSIONS}

The present paper dealt with the prediction of roll-waves occurrence in mud-flows. The spatial growth of a point-wise disturbance predicted by the linearized flow model of a Herschel and Bulkley fluid has been analyzed, in the neighborhood of a base initial uniform condition. The resulting growth-factor has been used to extend to mud-flows the minimum channel length criterion used for the prediction of roll-waves in clear-water flows. The comparison of the theoretical predictions with available experimental evidences has shown that the minimum channel length can allow only to partially justify the absence of roll-waves in unstable hypocritical conditions of flow in a Herschel and Bulkley fluid. This result suggests that, while in clear-water flows such a criterion is succesfully applied to predict the roll-waves occurence, its use in mud-flows does not guarantee a correct prediction. It can be argued that since in mud-flows the instabilty conditions are often hypocritical, the downstream boundary condition plays a non-negligible role on the spatial evolution of the disturbance.

\section{REFERENCES}

Ancey, C., 2001. Snow Avalanches. Selected topics in geological and geomorphological fluid mechanics. Berlin, Springer.

Ancey, C., 2005. Solving the Couette inverse problem by using a wavelet-vaguelette decomposition. J. Rheol., 49, 441-460.

Ancey, C., Andreini, N., Epely-Chauvin, G., 2012. Viscoplastic dambreak waves: Review of simple computational approaches and comparison with experiments. Advances in Water Resources, 48, 79-91. 
Bagnold, R.A., 1954. Experiments in a gravity-free dispersion of large solid spheres in a Newtonian fluid under shear. Proc. Royal Soc. London, Ser. A, 225, 49-63.

Balmforth, N.J., Liu, J.J., 2004. Roll waves in mud. J. Fluid Mech., 519, 33-54.

Berlamont, J.F., 1976. Roll-waves in inclined rectangular open channels. BHRA Fluid Engineering, Newcastle, Vol. A2.

Berlamont, J.F., Vanderstappen, N., 1981. Unstable turbulent flow in open channels. J. Hydr. Div., 107 (HY4), 427-449.

Bird, R.B., Dai, G.C., Yarusso, B.J., 1983. The rheology and flow of viscoplastic materials. Rev. Chem. Eng, 1, 1-70.

Brock, R.R., 1969. Development of roll-waves trains in open channels. J. Hydr. Div., 95 (HY4), 1401-1427.

Coussot, P., 1994. Steady, laminar, flow of concentrated mud suspensions in open channel. J. Hydr. Res., 32, 4, 535-559.

Coussot, P., 1997. Mudflow Rheology and Dynamics. Rotterdam, Balkema.

Dent, J.D., Lang, T.E., 1983. A biviscous modified Bingham model of snow avalanche motion. Annals Glaciology, 4, 4246.

Di Cristo, C., Vacca, A., 2005. On the convective nature of roll waves instability. J. Appl. Math., 3, 259-271.

Di Cristo, C., Iervolino, M., Vacca, A., Zanuttigh, B., 2008. Minimum channel length for roll-waves generation. J. Hydraul. Res., 46(1), 73-79.

Di Cristo, C., Iervolino, M., Vacca, A., Zanuttigh, B., 2009. Roll waves prediction in dense granular flows. J. Hydrol., 377(1-2), 50-58.

Di Cristo, C., Iervolino, M., Vacca, A., Zanuttigh, B., 2010. Influence of relative roughness and Reynolds number on the roll waves spatial evolution. Journal of Hydraulic Engineering, ASCE, 136(1), 24-33.

Di Cristo, C., Iervolino, M., Vacca, A., 2012. Green's function of the linearized Saint-Venant equations in laminar and turbulent flows. Acta Geophysica, 60(1), 173-190.

Di Cristo, C., Iervolino, M., Vacca, A., 2013a. Waves dynamics in a linearized mud-flow shallow model. Applied Mathematical Sciences, 7(8), 377-393.

Di Cristo, C., Iervolino, M., Vacca, A., 2013b. Boundary conditions effect on linearized mud-flow shallow model. Acta Geophysica, 61(3), 649-667.

Di Cristo, C., Iervolino, M., Vacca, A., 2013c. Gravity-Driven Flow of a Shear-Thinning Power-Law Fluid over a Permeable Plane. Applied Mathematical Sciences, 7(33), 1623-1641.

Di Cristo, C., Iervolino, M., Vacca, A., 2013d. Applicability of kinematic, diffusion and quasi-steady dynamic wave models to shallow mud flows. J. Hydrol. Eng., doi: 10.1061/(ASCE)HE.1943-5584.0000881.

Di Nucci, C., Russo Spena, A., 2011. Discussion of "Energy and momentum under critical flow conditions" by O. CastroOrgaz, J.V. Giráldez and J.L. Ayuso. J. Hydraul. Res. 49, 1, $127-130$.

Di Nucci, C., Russo Spena, A., Todisco, M.T., 2007. On the non-linear unsteady water flow in open channels. Il Nuovo Cimento B 122, 3, 237-255, doi: 10.1393/ncb/i2006-10174$\mathrm{x}$.

Fernández-Nieto, E.D., Noble, P., Vila, J.P., 2010. Shallow water equation for non-Newtonian fluids. J. Non-Newton Fluid. Mech., 165(7), 12-32.

Greco, M., Iervolino, M., Leopardi, A., Vacca, A., 2012. A two-phase model for fast geomorphic shallow flows. Int. J. Sed. Res., 27, 4, 409-425.
Huang, X., Garcia, M.H., 1997. A perturbation solution for Bingham-plastic mudflows. J. Hydr. Engrg., ASCE, 123, 11, 986-994.

Huang, X., Garcia, M.H., 1998. A Herschel-Bulkley model for mud flow down a slope. J. Fluid Mech., 374, 305-333.

Huerre, P., Monkewitz, P.A., 1990. Local and global instabilities in spatially developing flows. Annual Review of Fluid Mechanics, 22, 473-537.

Hwang, C., Chen, J., Wang, J., Lin, J., 1994. Linear stability of power law liquid film flows down an inclined plane. J. Phys. D., Appl. Phys., 27, 2297-2301.

Iverson, R.M., 1997. The physics of debris flows. Rev. Geophys., 35(3), 245-296.

Jackson, R., 2000. The dynamics of fluidized particles. Cambridge University Press, Cambridge.

Jiang, L., LeBlond, P.H., 1992. The coupling of a submarine slide and the surface water waves which it generates. J. Geophys. Res., 97(C8), 731-744.

Johnson, A.M, 1970. Physical Processes in Geology. Freeman, San Francisco.

Julien, P.Y., Hartley, D.M., 1986. Formation of roll waves in laminar sheet flow. J. Hydr. Res., 24(1), 5-17.

Laigle, D. Coussot, P., 1997. Numerical modeling of mudflows. J. Hydraul. Eng., 123, 617-623.

Liggett, J.A., 1975. Stability. Chap. 6 in Unsteady flow in open channel. Vol. 1. K. Mahmood, K., Yevjevich, V., (Eds.), Water Resources Publications, Fort Collins.

Liu, K.F., Mei, C.C., 1989. Slow spreading of a sheet of Bingham fluid on an inclined plane. J. Fluid Mech., 207, 505-529.

Mainali, A., Rajaratnam, N., 1994. Experimental study of debris flows. J. Hydr. Engrg., ASCE, 120, 1, 104-123.

Montuori, C., 1963. Discussion of "Stability aspect of flow in open channels". J. Hydr. Div., 89(HY4), 264-273.

Ng, C., Mei, C.C., 1994. Roll waves on a shallow layer of mud modelled as a power-law fluid. J. Fluid Mech., 263, 151184.

O’Brien, J.S., Julien, P.Y., 1988. Laboratory analysis of mudflow properties. J. Hydr. Engrg., ASCE, 114, 8, 877-887.

Pascal, J.P., 2006. Instability of power-law fluid flow down a porous incline. J. Non-Newtonian Fluid Mech., 133, 109120.

Pascal, J.P., D’Alessio, S.J.D., 2007. Instability of power-law fluid flows down an incline subjected to wind stress. Applied Mathematical Modelling, 31, 1229-1248.

Pitman, E.B., Le, L., 2005. A two-fluid model for avalanche and debris flows. Phil. Trans. R. Soc. A, 363, 1573-1601.

Ponce, V.M., Simon, D.B., 1977. Shallow water propagation in open channel flow. J. Hyd. Div., 103, 1461-1476.

Takahashi, T., 1978. Mechanical characteristics of debris flow. J. Hydr. Div., ASCE, 104, 8, 1153-1169.

Takahashi, T., 1991. Debris Flow. IAHR Monograph Series. Balkema, Rotterdam, The Netherlands. 165 pp.

Trowbridge, J.H., 1987. Instability of concentrated free surface flows. J. Geoph. Res., 92 (C9), 9523-9530.

Vedernikov, V.V., 1946. Characteristics feature of a liquid flow in open channel. USSR Academy of Science, 52, 207-210.

Zanuttigh, B., Lamberti, A., 2007. Instability and surge development in debris flows. Rev. Geophys., 45, RG3006, doi:10.1029/2005RG000175 (AGU Publ., USA).

Received 26 March 2013

Accepted 13 September 2013 